$>$ À partir du registre du cancer d'Eindhoven aux Pays-Bas, cette contribution fait le point sur les traitements et la survie du cancer du sein chez la femme âgée en analysant l'impact du pronostic et le traitement adjuvant en fonction des facteurs de comorbidité liés au vieillissement. <

\section{Patientes âgées atteintes de cancer du sein aux Pays-Bas : traitement et survie}

Marieke Louwman
Eindhoven a une population d'environ 200000 habitants. L'enregistrement des patientes atteintes de cancer du sein a débuté en 1955. Avec le temps, d'autres hôpitaux ont rejoint ce groupe. À ce jour, le registre tumoral d'Eindhoven couvre 2,4 millions de personnes. Depuis 1989, la totalité des Pays-Bas sont couverts par neuf centres régionaux anticancéreux. Tous ces centres travaillent de la même manière. Ils enregistrent chaque tumeur survenant aux Pays-Bas. Ces enregistrements se font à partir des données cliniques et anatomopathologiques et des bilans de sortie des hôpitaux. La saisie est réalisée dans les centres anti-cancéreux. Les employés recueillent les données telles que l'âge, le sexe, l'adresse, la date d'incidence, la localisation de la tumeur, la morphologie, le type histologique, le stade, et le traitement initial. Celles-ci ne comprennent pas le traitement ultérieur. Chaque année, nous essayons de savoir si les personnes sont encore en vie. Nous disposons donc d'un suivi des malades.

La probabilité pour un patient d'avoir d'autres maladies au moment du diagnostic du cancer augmente avec l'âge. D'où une prise en charge du cancer plus complexe. La mise en évidence d'une comorbidité permet de distinguer les patients atteints de cancer et en meilleure santé des plus faibles. Les patients âgés sont souvent exclus des essais cliniques. Par conséquent, il est difficile de savoir si les directives de traitement sont adaptées aux patients âgés.

Nous définissons la comorbidité comme l'existence de maladies graves concomitantes et présentes au moment du diagnostic du cancer. Celles-ci ont été

Le registre tumoral d'Eindhoven est un exemple pour tous les registres tumoraux dans le monde.

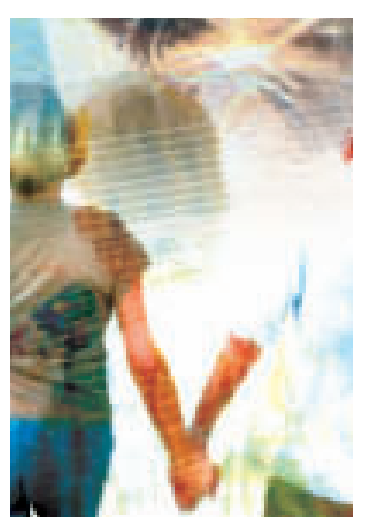

Médecin épidémiologiste au Registre du Cancer d'Eindhoven, Eindhoven Cancer Registry, Comprehensive Cancer Centre South, P0 Box 231, $\mathrm{NL}-5600$ AE Eindhoven, Pays-Bas. m.louwman@ikz.nl

enregistrées depuis 1993 dans le registre tumoral d'Eindhoven. Ces données sont aussi recueillies en même temps que celles concernant la tumeur dans les dossiers médicaux par le personnel chargé del'enregistrement. Pour s'assurer que les données sont précises, nous avons réalisé plusieurs études de validation. Parmi les conditions enregistrées figurent les BPCO (bronchopneumopathies chroniques obstructives), les maladies cardiovasculaires, les maladies cérébrovasculaires, les antécédents de tumeur, l'hypertension et le diabète.

En 1995, l'exhaustivité de l'enregistrement de la comorbidité a été évaluée. Elle a montré quelques insuffisances pour les maladies cardiovasculaires. Cela est dû à la difficulté pour le personnel à identifier la maladie à partir des dossiers médicaux. Une amélioration a été apportée par l'ajout des noms des maladies et l'explication des acronymes. Des instructions supplémentaires ont aussi été fournies à l'équipe. Depuis 1995, l'exhaustivité a été améliorée.

Pour l'ensemble des tumeurs, environ $30 \%$ des patients masculins n'ont aucune comorbidité. Pour les femmes, ce chiffre est plus faible. Environ $10 \%$ des hommes avaient déjà eu une tumeur avant le diagnostic du cancer. La BPCO et les maladies cardiovasculaires sont très prévalentes chez les hommes. L'hypertension est très prévalente chez les femmes. 
Parmi les patients jeunes, de 50 à 60 ans, $50 \%$ présentent une comorbidité. Parmi les patients âgés, $4 / 5$ des patients atteints de cancer, c'est-à-dire $80 \%$, ont d'autres maladies graves.

La prévalence de la BPCO et des maladies cardiovasculaires est élevée parmi les patients atteints de cancer du poumon. La prévalence de comorbidité est plus faible chez les patientes atteintes de cancer du sein que chez les femmes atteintes d'autres tumeurs.

Les Pays-Bas ont une incidence et un taux de mortalité élevés pour le cancer du sein (Figure 1). L'incidence et la mortalité augmentent avec l'âge. Aux âges moyens, le cancer du sein est une cause importante de décès chez les femmes. Une femme sur cinq décédant à un âge moyen meurt de cancer du sein.

Parmi les femmes pour lesquelles un diagnostic de cancer du sein est posé avant l'âge de 50 ans, près de $80 \%$ n'ont pas d'autre maladie grave au moment du diagnostic. Ce pourcentage diminue avec l'âge. Soixante-cinq pour cent des patientes de plus de 80 ans ont une ou plusieurs autres maladies graves. Ces maladies comprennent un précédent cancer, une maladie cardiovasculaire, une BPCO, un diabète, une maladie cérébrovasculaire ou des troubles du tractus digestif.

Le nombre des patientes traitées uniquement par chirurgie augmente avec l'âge. Le nombre des patientes traitées sans radiothérapie et avec une thérapie systémique augmente également. L'utilisation d'une thérapie systémique augmente et la radiothérapie diminue avec l'âge. Toutes les patientes âgées de moins de 50 ans ayant subi une chirurgie mammaire conservatrice ont également subi une exploration axillaire et une radiothérapie. Après 50 ans, la proportion de femmes recevant un traitement complet diminue: l'exploration axillaire et la radiothérapie ne sont pas systématiquement pratiquées.

Le nombre de patientes atteintes de cancer du sein de stade I recevant une radiothérapie décroît avec l'âge. La probabilité de bénéficier d'une radiothérapie au-delà de 80 ans représente

\section{Cancer du sein : taux brut (pour 100,000)(âge 65-75+)}

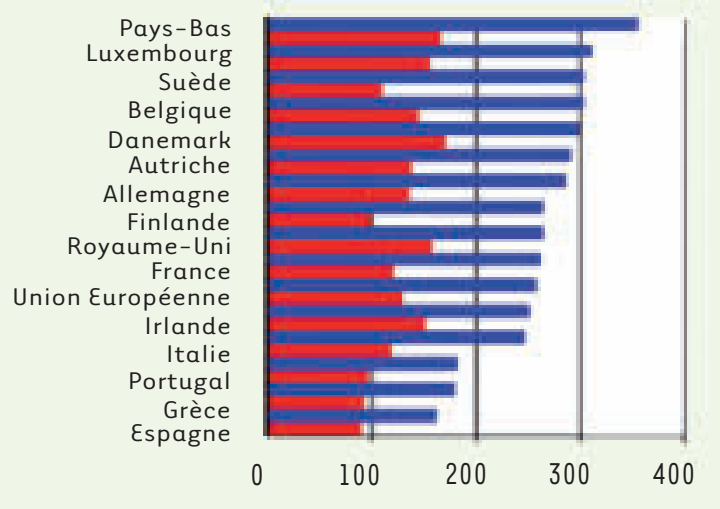

Incidence

Mortalité

Cancer dans I'Union Européenne (1995), IARC, 1999
$40 \%$ de la même probabilité à moins de 60 ans. Plus une patiente dispose de comorbidités, plus faibles sont ses chances de recevoir une radiothérapie. Mais le rôle de l'âge est plus important que celui de la comorbidité : plus l'âge augmente et moins la chirurgie mammaire conservatrice est suivie d'une radiothérapie. Ni l'âge ni la comorbidité n'ont affecté la dose ou le nombre de séances pour les malades ayant eu une radiothérapie. L'incidence des complications pour les patientes âgées et jeunes reste la même.

Quelle est l'incidence de la comorbidité sur l'évolution? Les malades sans comorbidité dans la tranche d'âge 50-70 ans ont une survie relative à cinq ans de $87 \%$. Celles sans comorbidité dans la tranche d'âge > à 70 ans ont une survie relative à cinq ans de $93 \%$. Ce taux de survie diminue avec l'augmentation des comorbidités. Les patientes ayant eu antérieurement un cancer ont une survie plus faible que celles qui sont atteintes d'une maladie cardiovasculaire avant le diagnostic du cancer. Parmi les patientes de plus de 70 ans, le taux de survie est plus faible chez celles qui sont atteintes de diabète.

Dans une analyse rapportée à l'âge et au traitement, le risque de mourir d'une maladie cardiovasculaire est de 1,8 . Le rapport de risque est de 1,5 dans la tranche d'âge 65-80 ans et de 3,5 dans le groupe de plus de 80 ans. Les effets de la comorbidité sont plus faibles. $\varepsilon n$ présence d'une comorbidité, le risque est de presque 1,5. Lorsqu'il existe deux maladies, le risque est deux fois plus élevé.

$\varepsilon n$ résumé, il est possible dans les renseignements de prendre en compte la comorbidité et

son enregistrement est rapide. Nos résultats montrent que la comorbidité augmente avec l'âge. Le type de maladies est nettement différent selon la tumeur et le sexe du patient (Figure 2). La présence de comorbidité conduit généralement à un traitement moins agressif. Pour le cancer du sein, cela signifie moins de radiothérapie adjuvante, moins de chimiothérapie et davantage de thérapie hormonale. Le pronostic est indé-

Figure 1. Incidence et mortalité par cancer du sein en Europe. 
pendant de l'âge. C'est également le cas de la comorbidité. L'impact du pronostic ne peut être expliqué uniquement par le traitement. Le traitement moins agressif des patients âgés n'est pas obligatoirement justifié. Il est nécessaire de distinguer entre les personnes qui sont en bonne santé et celles qui sont fragiles. Des données prospectives pourraient permettre de savoir pourquoi les patients âgés ont été traités de manière moins agressive, en

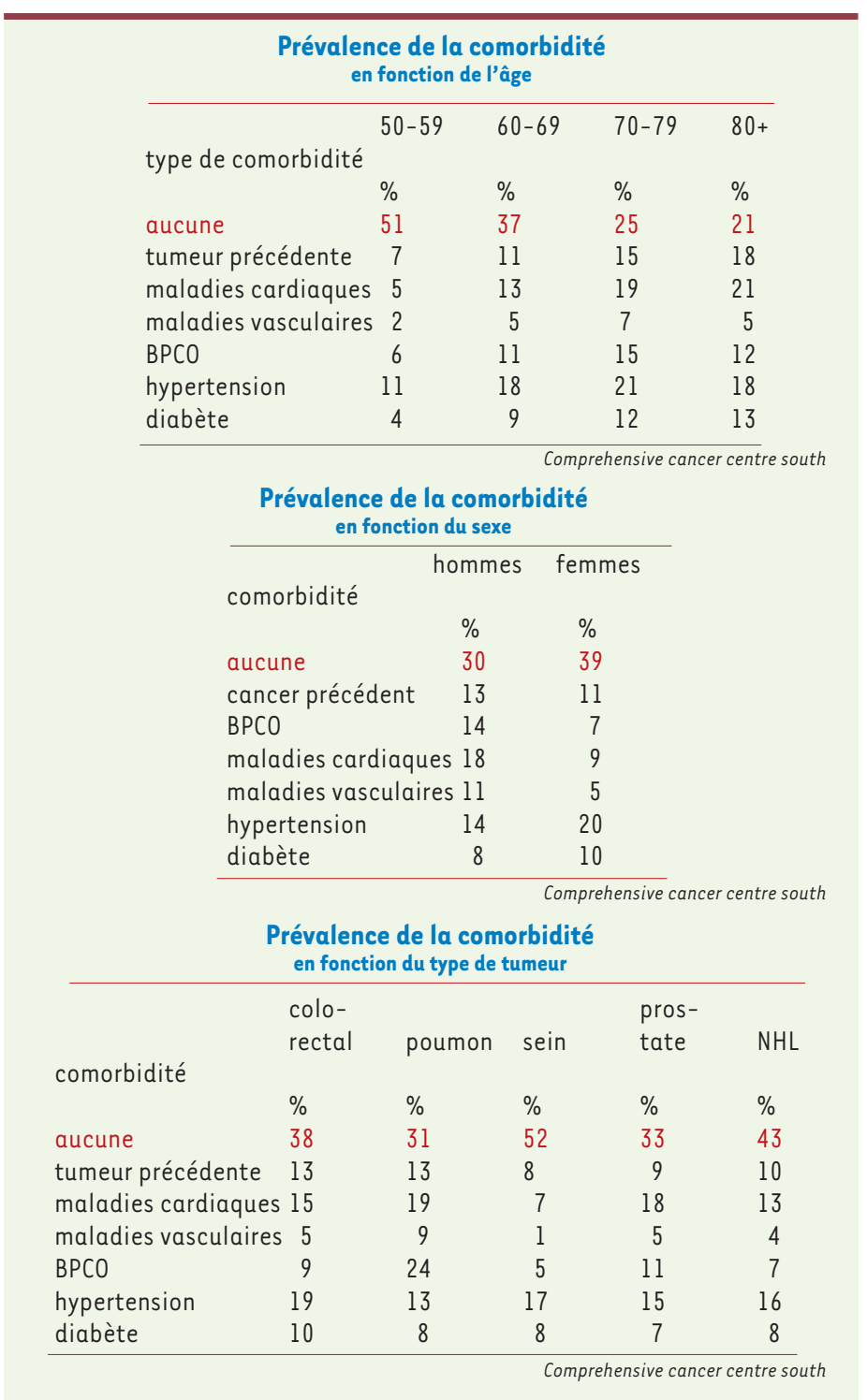

Figure 2. Prévalence des comorbidités associées aux cancers en fonction de l'âge, du sexe et de la pathologie cancéreuse. NHL : non-Hodgkin lymphoma. particulier si le type de traitement résulte du choix du patient ou du médecin traitant. Il serait également intéressant d'analyser les facteurs sociaux, psychologiques et de qualité de vie pour en tirer des conclusions plus complètes. De même, il serait intéressant d'analyser le pronostic, par rapport à la toxicité du traitement ou la qualité de vie. Les complications ayant suivi le traitement ont été étudiées pour un sous-groupe de patients. Mais nous ne pouvons affirmer qu'elles sont applicables à tous les patients âgés. Si les patients âgés étaient traités selon les mêmes directives, seraient-ils atteints de davantage de complications? Il est nécessaire d'adapter un traitement individualisé pour les patients âgés atteints de cancer avec une comorbidité. $\diamond$

\section{SUMMARY}

Elderly breast cancer patients in The Netherlands: treatment and survival

From the Eindhoven cancer registry in the Netherlands, this contribution reviews treatments and survival of the breast cancer at the old woman by analyzing the impact of the forecast and the additive treatment according to the factors of comorbidity bound to the ageing. $\diamond$

\section{RÉFÉRENCES}

1. Louwman WJ, Janssen-Heijnen ML, Houterman S, et al. Less extensive treatment and inferior prognosis for breast cancer patient with comorbidity : a population-based study. Eur J Cancer $2005 ; 41$ : 779-85.

2. Coebergh JW, Janssen-Heijnen ML, Post PN, Razenberg PP. Serious comorbidity among unselected cancer patients newly diagnosed in the southeastern part of The Netherlands in 1993-1996. J Clin Epidemiol $1999 ; 52$ : 1131-6.

3. Van de Poll-Franse LV, Houterman S, Janssen-Heijnen ML, et al. Less aggressive treatment and worse overall survival in cancer patients with diabetes: a large population based analysis. Int J Cancer 2007 ; $120: 1986-92$.

4. Janssen-Heijnen ML, Houterman S, Lemmens VE, et al. Age and comorbidity in cancer patients: a population-based approach. Cancer Treat Res $2005 ; 124: 89-107$.

5. Janssen-Heijnen ML, Houterman S, Lemmens VE, et al. Prognostic impact of increasing age and co-morbidity in cancer patients: a populationbased approach. Crit Rev Oncol Hematol 2005 ; 55 : 231-40.
TIRÉS À PART

M. Louwman 